\title{
Evaluación de tres dosis de un preparado microbiano, obtenido en Ecuador, en la respuesta productiva y sanitaria de cerdos en posdestete
}

\author{
Three doses evaluation of a microbial preparation \\ obtained in Ecuador, in the productive and sanitary \\ response of post-weaning pigs
}

Luis Gerardo Flores-Mancheno ${ }^{1,}$ Yaneisy García-Hernández²

Fredy Bladimir Proaño-Ortiz ${ }^{3,}$

William Orlando Caicedo-Quinche ${ }^{4}$

\begin{abstract}
Resumen

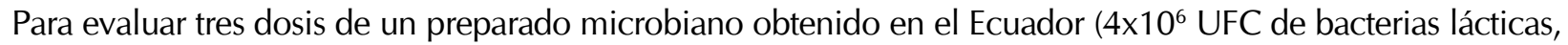
$1,5 \times 10^{5}$ UFC de levaduras por cada mililitro), en el comportamiento productivo y de salud en cerdos durante la etapa post-destete, se aplicó un diseño completamente aleatorizado, con cuatro tratamientos y cuatro repeticiones por tratamiento: T1 concentrado, T2, T3, T4 (concentrado más $5 \mathrm{~mL} / \mathrm{kg}$ de peso vivo (PV), $10 \mathrm{~mL} / \mathrm{kg}$ PV 15 $\mathrm{mL} / \mathrm{kg}$ PV del preparado microbiano, respectivamente). Se utilizaron 160 cerdos castrados del cruce Landrace $x$ Large White con Blanco Belga x Pietrain, de $28 \mathrm{~d}$ de edad y con 6,99 kg de PV. El mayor peso vivo final y la mejor ganancia de peso total y diaria $(\mathrm{P}<0,0001)$ se obtuvo en el grupo de cerdos donde se agregó $15 \mathrm{~mL}$ $\mathrm{kg}$ PV del preparado microbiano, con valores de $25,78 \mathrm{~kg}, 18,78 \mathrm{~kg}$ y $447,25 \mathrm{~g}$, respectivamente. En el mismo grupo experimental se encontraron las conversiones de materia seca, proteína bruta y energía metabolizable más eficientes $1,54 \mathrm{~kg} / \mathrm{kg}$ PV y aumento de peso vivo de 383,92 g/kg PV y 23,16 MJ/kg PV, respectivamente. También en este grupo se registró la menor presencia de animales con diarrea $(P<0,0001) 6,85 \%$. Se concluye que la adición de $15 \mathrm{~mL} / \mathrm{kg}$ PV mejora el comportamiento productivo y sanitario de cerditos destetados.
\end{abstract}

Palabras Clave: peso final, conversión de materia seca, animales con diarrea.

\section{Abstract}

1 Ph.D. Escuela Superior Politécnica de Chimborazo (Riobamba, Ecuador). Iflores@espoch.edu.ec.

2 Ph.D. Instituto de Ciencia Animal (Mayabeque, Cuba). yaneisyg@ica.co.cu.

3 Ph.D. Escuela Superior Politécnica de Chimborazo (Riobamba, Ecuador). fproano@espoch.edu.ec.

4 Ph.D. Universidad Estatal Amazónica (Pastaza, Ecuador). cienciasdelatierra@uea.edu.ec. 
To evaluate three doses of a microbial preparation obtained in Ecuador $\left(4 \times 10^{6} \mathrm{CFU}\right.$ of lactic bacterias, $1.5 \times 10^{5} \mathrm{CFU}$ of yeast per milliliter), in the productive and health performance in pigs during the post-weaning stage, a completely randomized design was used, with four treatments and four replicates per treatment: T1 concentrated, T2, T3, T4 (concentrate plus $5 \mathrm{~mL} / \mathrm{kg}$ of body weight (BW), $10 \mathrm{~mL} / \mathrm{kg}$ BW, $15 \mathrm{~mL} / \mathrm{kg}$ BW of microbial preparation, respectively). 160 barrows of the crossing Landrace $x$ Large White with White Belgian x Pietrain, of 28 days of age and $6.99 \mathrm{~kg}$ of BW were used. The higher final body weight and the best gain of total and daily weight $(P<0.0001)$ was obtained in the group of pigs in which $15 \mathrm{~mL} / \mathrm{kg}$ BW of microbial preparation was added, with values of $25.78 \mathrm{~kg}$, $18.78 \mathrm{~kg}$ and 447.25 $\mathrm{g}$, respectively. In the same experimental group more efficient conversions of dry matter, crude protein and metabolizable energy were found, $1.54 \mathrm{~kg} / \mathrm{kg}$ BW and gain in body weight of $383.92 \mathrm{~g} / \mathrm{kg}$ BW and $23.16 \mathrm{MJ} / \mathrm{kg} \mathrm{BW}$, respectively. Also in this group the least presence of animals with diarrhea $(\mathrm{P}<0,0001)$ $6.85 \%$ was recorded. It is concluded that the addition of $15 \mathrm{~mL} / \mathrm{kg} \mathrm{BW}$ improves the productive and sanitary performance of weaned piglets.

Keywords: final weight, conversion of dry matter, animals with diarrhea. 


\section{Introducción}

El destete impone un gran estrés en lechones y se acompaña de cambios fisiológicos, microbiológicos e inmunológicos en el tracto gastrointestinal (1). Debido a estos cambios, el período después del destete se caracteriza por una alta incidencia de trastornos intestinales con diarrea y bajo rendimiento del crecimiento de los animales $(2,3)$.

La necesidad de controlar las patologías digestivas en los sistemas intensivos de producción de cerdos ha conllevado a la utilización masiva de antibióticos como aditivos alimenticios (4). Sin embargo, el empleo de productos antimicrobianos en dosis bajas en la alimentación de animales que se destinan al consumo humano, con el propósito de mejorar el crecimiento y la prevención de enfermedades, se asocia a la crisis de salud global relacionada con la resistencia a los antimicrobianos. En el ámbito internacional, varias jurisdicciones han respondido al restringir el uso de estos productos (5).

La utilización de probióticos, como aditivos microbianos vivos, es una alternativa que aporta beneficios a la salud del animal, por cuanto mejora el equilibrio microbiano intestinal. La aplicación de probióticos en la alimentación de cerdos ha permitido mejorar los indicadores. Los problemas fundamentales que se presentan con el uso de probióticos se centran en los altos precios de estos productos, la viabilidad de los microorganismos y la variabilidad de los resultados de su aplicación en los animales (6).

En los sistemas productivos de Ecuador no se emplean ampliamente los aditivos microbianos. Al parecer en los últimos cinco años comienzan a realizarse evaluaciones y se informan trabajos investigativos donde se evidencian las ventajas del uso de estos productos $(7,8,9)$.

En este sentido Díaz (10) informó la obtención de un preparado microbiano nativo, a partir de suero fresco de leche, con alta concentración de bacterias lácticas y levaduras, además de contener ácidos orgánicos y enzimas. Características que resultaron de interés para su aplicación en la obtención de bio-productos destinados a la alimentación ani- mal y que le confieren potencialidades para su uso como aditivos alimentarios.

De ahí que el objetivo de esta investigación fue determinar el efecto de tres dosis del preparado microbiano ecuatoriano en la respuesta productiva y sanitaria de cerdos en post destete.

\section{Materiales y métodos}

Localización del experimento. El experimento se desarrolló en la Unidad Académica Porcina de la Facultad de Ciencias Pecuarias (FCP) de la Escuela Superior Politécnica de Chimborazo (ESPOCH), Ecuador.

Procedimiento experimental. Se utilizaron 160 cerdos castrados, cruce Landrace-Large White $x$ Belga-Pietrain, de $28 d$ de edad y con 6,99 $\pm 0,18$ kg. Cada unidad experimental se compuso de 10 cerdos alojados en corrales colectivos de $2 \times 2,25$ $\mathrm{m}$ y piso de cemento, con densidad de un cerdo por $0,45 \mathrm{~m} 2$. Los animales recibieron cuatro tratamientos experimentales, bajo un diseño completamente aleatorizado. El alimento se ofreció 2 veces por día a las 8:00 am y 4:00 pm, el consumo de alimento se detalla en la tabla 1 . El agua se suministró ad libitum en bebederos tipo tetina.

Tabla 1. Consumo de alimento promedio en la etapa de post destete.

\begin{tabular}{|c|c|c|}
\hline $\begin{array}{c}\text { Edad de los } \\
\text { animales (días) }\end{array}$ & $\begin{array}{c}\text { Consumo } \\
\text { promedio (kg/ } \\
\text { día) }\end{array}$ & $\begin{array}{c}\text { Consumo } \\
\text { semanal } \\
\mathbf{( k g})\end{array}$ \\
\hline $28-34$ & 0,38 & 2,66 \\
\hline $35-41$ & 0,55 & 3,85 \\
\hline $42-48$ & 0,70 & 4,90 \\
\hline $49-55$ & 0,85 & 5,95 \\
\hline $56-62$ & 0,99 & 6,90 \\
\hline $63-69$ & 1,12 & 7,85 \\
\hline $\begin{array}{c}\text { Consumo total de } \\
\text { la etapa }\end{array}$ & & $\mathbf{3 2 , 1 1}$ \\
\hline
\end{tabular}

Los tratamientos $(\mathrm{T})$ evaluados fueron: 
T1: concentrado sin aditivo

T2: concentrado $+5 \mathrm{~mL}$ del preparado micro-

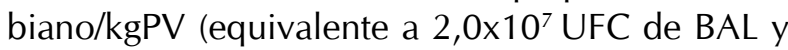
$7,5 \times 10^{5}$ UFC de levaduras)

T3: concentrado $+10 \mathrm{~mL}$ del preparado micro-

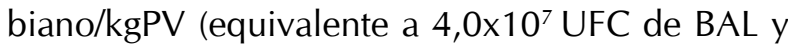
$1,5 \times 10^{6}$ UFC de levaduras)

T4: concentrado $+15 \mathrm{~mL}$ del preparado microbiano/kgPV (equivalente a 6,0×107 UFC de BAL y $2,25 \times 10^{6}$ UFC de levaduras).

El preparado de bacterias lácticas y levaduras empleado como aditivo, en el estudio en la categoría de posdestete se elaboró según la metodología descrita por (10).

El preparado presentó valores similares a los informados por (10): $4 \times 10^{6}$ UFC de bacterias lácticas, $1,5 \times 10^{5}$ UFC de levaduras por cada mililitro de producto y $\mathrm{pH}$ de 3,9 . A partir de estos valores se calcularon las concentraciones microbianas a evaluar en cada experimento.

Las materias primas para la elaboración de los balanceados se adquirieron en el mercado local de la ciudad de Riobamba. Éstos se formularon según los requerimientos nutricionales (11) para cerdos en la etapa post destete. Su composición química y aportes calculados se muestran en las tablas 2 y 3 , respectivamente.

Tabla 2. Composición de las dietas base utilizadas en cerdos en post destete

\begin{tabular}{|l|c|}
\hline Ingrediente & \% \\
\hline Maíz (\%) & 35,00 \\
\hline Polvillo de arroz (\%) & 5,00 \\
\hline Afrecho de trigo (\%) & 9,70 \\
\hline Harina de soya (\%) & 35,20 \\
\hline Harina de pescado (\%) & 2,00 \\
\hline DL-Metionina (\%) & 0,40 \\
\hline
\end{tabular}

\begin{tabular}{|l|c|}
\hline Ingrediente & \% \\
\hline L-Lisina (\%) & 0,40 \\
\hline Palmiste (\%) & 5,00 \\
\hline Melaza (\%) & 2,00 \\
\hline Aceite de palma (\%) & 2,90 \\
\hline Carbonato de calcio $\%$ ) & 0,50 \\
\hline Fosfato dicálcico (\%) & 1,10 \\
\hline Cloruro de sodio (\%) & 0,50 \\
\hline Pecutrín ${ }^{\circledR 1}(\%)$ & 0,30 \\
\hline
\end{tabular}

${ }^{1}$ Pre-mezcla mineral y vitamínica (Ecuaquímica 2000)

Tabla 3. Composición química calculada de las dietas experimentales (90\% MS).

\begin{tabular}{|l|c|}
\hline Nutriente & Aporte \\
\hline Proteína bruta (\%) & 22,49 \\
\hline Lisina (\%) & 1,22 \\
\hline Metionina + Cistina (\%) & 0,69 \\
\hline Triptófano (\%) & 0,21 \\
\hline Calcio (\%) & 0,71 \\
\hline Fósforo (\%) & 0,81 \\
\hline Fósforo disponible (\%) & 0,42 \\
\hline Extracto etéreo (\%) & 5,40 \\
\hline Fibra bruta (\%) & 4,84 \\
\hline Energía metabolizable (MJ/kg) & 13,60 \\
\hline
\end{tabular}

El preparado microbiano se aplicó en el concentrado según el tratamiento establecido. Para esto se determinó el peso promedio total de cada corral y se ajustó semanalmente la cantidad de preparado a añadir en la dieta. La mezcla de ambos se realizó en recipientes de plástico para garantizar la homogenización.

La respuesta de los animales destetados al suministro de las tres dosis del aditivo en las dietas se determinó a través de los siguientes indicadores: peso vivo promedio de cada corral a los 70 días 
de edad, número de animales que presentaron diarreas diariamente, número de animales muertos por tratamiento y causas de las diarreas. También, se calculó la ganancia de peso de la etapa; ganancia de peso diaria; conversión de materia seca, proteína bruta y energía metabolizable; porcentajes de animales con diarreas y la mortalidad.

Las diarreas se clasificaron en producidas por Salmonella, Escherichia coli y trastornos digestivos. Para la detección de Salmonella, se sembraron muestras de heces en agar Salmonella-Shigella (12), las placas se incubaron $18-24 \mathrm{~h}$ a $35 \pm 2{ }^{\circ} \mathrm{C}$ y se contaron las colonias negativas que son incoloras (lactosa negativa), las colonias positivas son de color rosa-rojo (lactosa-positiva) y las colonias de centro negro productoras de $\mathrm{H}_{2} \mathrm{~S}$. Para la detección de $E$. coli, se realizaron siembras en placas (13). Estas se incubaron durante $24-48 \mathrm{~h}$ a $42{ }^{\circ} \mathrm{C}$ y se contaron las colonias positivas que aparecieron de color azul o rojo-azul. Las diarreas por trastornos digestivos se determinaron por diferencia entre el número total de muestras analizadas menos las que dieron positivos para Salmonella y E. coli.

Análisis estadístico. Los datos experimentales se procesaron con el paquete estadístico InfoStat (14). Con el objetivo de conocer si existía influencia del peso inicial en el comportamiento productivo de los animales, se realizó análisis de covariable, donde se tomó el peso inicial como variable con- comitante. Se realizó análisis de varianza según diseño completamente aleatorizado y, en los casos necesarios, se aplicó la dócima de comparación de Duncan (15) para discriminar diferencias entre medias a $P<0,05$. A la variable de conteo y a las expresadas en porcentaje (diarreas y número de muertes), se les realizó análisis de comparación de proporciones (chi cuadrado) con el paquete estadístico ComparPro (16).

\section{Resultados y discusión}

La tabla 4 presenta los indicadores productivos obtenidos durante el experimento. Hubo diferencias entre tratamientos $(P<0,0001)$ para todas las variables en estudio excepto el peso inicial que fue la variable concomitante. Con respecto al control, el peso vivo final, la ganancia de peso total y la ganancia de peso diaria incrementaron en todos los tratamientos donde se incluyó el aditivo microbiano. Estos indicadores aumentaron al elevar la dosis del preparado microbiano y fue superior donde se adicionó $15 \mathrm{~mL} / \mathrm{kgPV}$. El incremento para la ganancia de peso total fue de 5,$66 ; 3,82$ y $1,10 \mathrm{~kg}$ respecto al control para la dosis de 15 $\mathrm{mL}$ del preparado/kgPV, $10 \mathrm{~mL} / \mathrm{kgPV}$ y $5 \mathrm{~mL} / \mathrm{kgPV}$, respectivamente. De manera similar, la ganancia de peso diaria mostró incrementos, en relación al control, de 134,25 g en el grupo tratado con 15 $\mathrm{mL} / \mathrm{kgPV}, 90 \mathrm{~g}$ en el de $10 \mathrm{~mL} / \mathrm{kgPV}$ y $26 \mathrm{~g}$ en el de $5 \mathrm{~mL} / \mathrm{kgPV}$. 
Tabla 4. Comportamiento productivo de cerdos en post destete al suministrar tres dosis de un preparado microbiano.

\begin{tabular}{|c|c|c|c|c|c|}
\hline \multirow{2}{*}{ Indicador } & \multirow{2}{*}{ Control } & \multicolumn{3}{|c|}{ Preparado microbiano, $\mathrm{mL} / \mathrm{kgPV}$} & \multirow{2}{*}{ EE $( \pm)$ Signif. } \\
\hline & & 5 & 10 & 15 & \\
\hline Peso inicial (kg) & 6,97 & 6,98 & 7,00 & 7,00 & $\begin{array}{c}0,02 \\
P=0,6442\end{array}$ \\
\hline Peso final (kg) & $20,12^{\mathrm{a}}$ & $21,22^{\mathrm{b}}$ & $23,94^{\mathrm{c}}$ & $25,78^{d}$ & $\begin{array}{c}0,17 \\
P<0,0001\end{array}$ \\
\hline Ganancia de peso total (kg) & $13,15^{\mathrm{a}}$ & $14,24^{\mathrm{b}}$ & $16,93^{c}$ & $18,78^{\mathrm{d}}$ & $\begin{array}{c}0,17 \\
P<0,0001\end{array}$ \\
\hline Ganancia de peso diaria $(\mathrm{g} / \mathrm{d})$ & $313,00^{\mathrm{a}}$ & $339,00^{b}$ & $403,00^{c}$ & $447,25^{d}$ & $\begin{array}{c}4,17 \\
P<0,0001\end{array}$ \\
\hline Conversión de MS (kg/kgPV) & $2,20^{d}$ & $2,03^{c}$ & $1,71^{\mathrm{b}}$ & $1,54^{\mathrm{a}}$ & $\begin{array}{c}0,02 \\
P<0,0001\end{array}$ \\
\hline Conversión de PB (g/kgPV) & $546,85^{d}$ & $508,85^{c}$ & $427,79^{b}$ & $383,92^{\mathrm{a}}$ & $\begin{array}{c}5,23 \\
P<0,0001\end{array}$ \\
\hline Conversión de EM (MJ/kgPV) & $33,01^{\mathrm{d}}$ & $30,64^{c}$ & $25,76^{\mathrm{b}}$ & $23,16^{\mathrm{a}}$ & $\begin{array}{c}0,32 \\
P<0,0001\end{array}$ \\
\hline
\end{tabular}

$a, b, c, d$ valores con letras no comunes en la misma fila difieren a $\mathrm{P}<0,05$ (Duncan 1955)

La conversión de la materia seca, proteína bruta y energía metabolizable mejoró respecto al control con la inclusión del preparado microbiano (tabla 3). Estos indicadores disminuyeron en la medida que se incrementó la dosis, con el mejor valor para el grupo de animales tratados con $15 \mathrm{~mL} / \mathrm{kgPV}$.

El comportamiento de los indicadores anteriores al adicionar el preparado microbiano en la dieta de los cerdos destetados y al incrementar su dosis, pudiera estar relacionado directamente con las concentraciones de microorganismos empleadas, que es uno de los factores que pueden incidir en la respuesta de los animales (17). Los microorganismos al ser ingeridos deben resistir la acidez gástrica y las sales biliares, que son las primeras barreras que limitan su supervivencia en el ecosistema gastrointestinal (18). De ahí que, al suministrar concentraciones superiores, los microorganismos tienen mayores posibilidades de sobrevivir y poder ejercer, posteriormente, su actividad probiótica. Las concentraciones empleadas en el presente experimento se encontraron en el rango sugerido por la (19), quienes plantearon que los probióticos deben tener una concentración de $10^{6}-10^{7}$ células $\cdot \mathrm{mL}^{-1} \mathrm{o}$ $\mathrm{g}^{-1}$ para garantizar su eficacia.

El preparado evaluado posee varias especies de Lactobacillus, según lo informado por (10), y la mayoría de estas se emplearon como probióticos por otros autores $(20,21,22)$. Por ejemplo, Lactobacillus casei cepa Shirota fue ampliamente estudiada y se describió como agente probiótico al ser capaz de colonizar el epitelio intestinal (23), estimular la respuesta inmune y prevenir infecciones enterobacterianas (24). También, LactobaciIlus rhamnosus se empleó como aditivo en cerdos lactantes y destetados por (25). Estos autores observaron una mejora de los indicadores de salud en el grupo tratado con relación al control, lo que influyó en una mejor expresión del potencial de crecimiento y salud de los cerdos jóvenes. A pesar de los efectos beneficiosos que pueden ejercer las especies de Lactobacillus, debe señalarse que éstos no se pueden atribuir de forma generalizada a un género o especie $(26,27)$, por lo que es imprescindible evaluar cada producto que se emplee con fines probióticos. 
Las levaduras, como se conoce, también se emplean en preparaciones probióticas y sus efectos pueden depender de la cepa o especie evaluada. Según (28), las levaduras no colonizan el tracto digestivo pero pueden estimular las disacaridasas de las microvellosidades del tracto gastrointestinal, estimular la inmunidad innata e inducir efectos antiadhesivos y antagonistas frente a patógenos, que se traducen en la mejora de los rendimientos productivos de especies monogástricas. Probablemente, las levaduras presentes en el preparado microbiano obtenido en Ecuador ejercieran estas acciones e incidieran en la respuesta positiva de los cerditos.
Relacionado con lo anterior, el preparado microbiano obtenido en Ecuador y las dosis utilizadas mejoraron la salud y redujeron la presentación de diarreas en los cerdos en post destete como se refleja en la figura 1. Se observa que hubo diferencias $(P<0,0001)$ entre tratamientos y que el preparado microbiano redujo el número de animales con diarreas. Respuesta que fue mayor con la dosis de $15 \mathrm{~mL} / \mathrm{kgPV}$. La diferencia entre el porcentaje de animales con diarreas de este tratamiento con el control fue de 41,15 unidades porcentuales. Valor que indica que la ingestión del aditivo mejoró la salud intestinal de los cerditos, lo que pudo estar relacionado con su efecto en la modulación del sistema inmune y la reducción o supresión de microorganismos potencialmente patógenos.

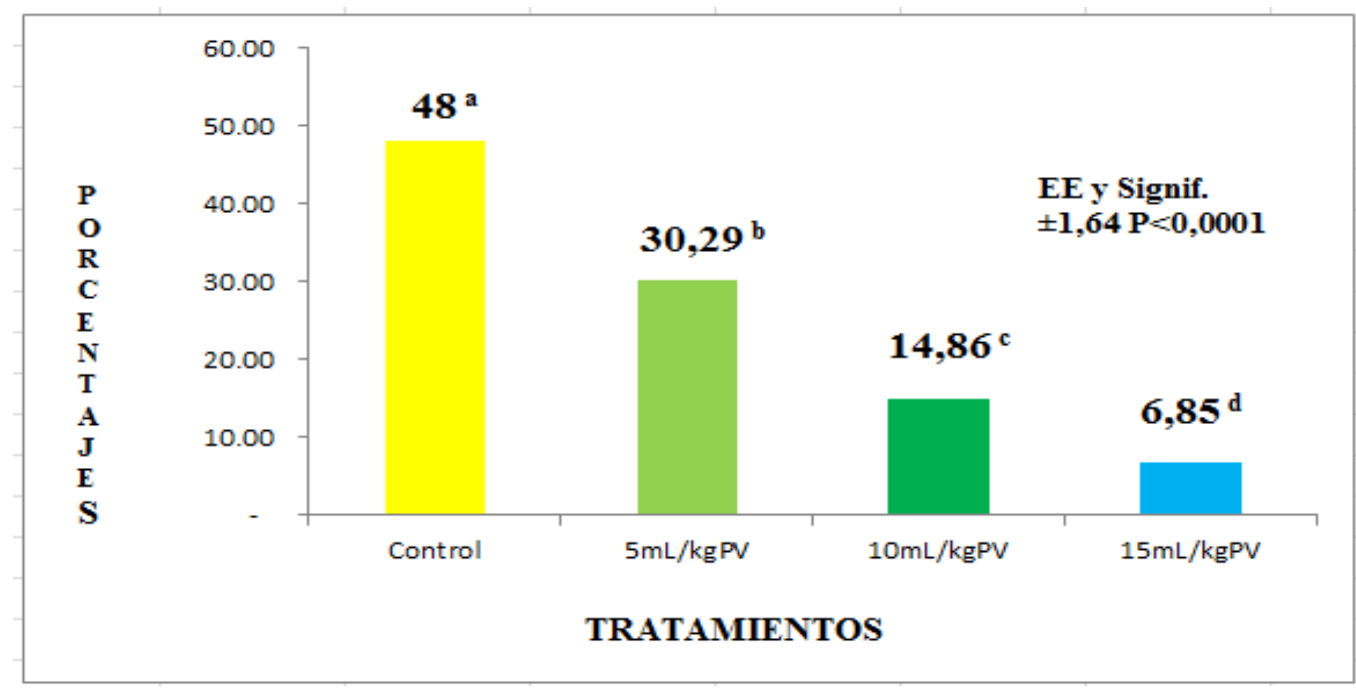

$a, b, c, d$ valores con letras no comunes difieren a $\mathrm{P}<0,05$ (Duncan 1955)

Figura 1. Porcentaje de animales con diarreas al incluir en la dieta tres dosis del preparado microbiano.

El mayor número de diarreas se produjo a causa de problemas digestivos, seguido de procesos infecciosos por E. coli y por último por Salmonella. Estos resultados, el porcentaje por cada causa y el número total de diarreas, se muestran en la tabla 5. En cuanto a las diarreas causadas por E. coli y problemas digestivos, hubo diferencias entre tratamientos $(\mathrm{P}<0,0001)$. Las causadas por Salmonella spp. difirieron entre el control y donde se incluyó una dosis de 10 ó $15 \mathrm{~mL} / \mathrm{kgPV}$. Este último tratamiento difirió también con el de $5 \mathrm{~mL} / \mathrm{kgPV}$. En todos los casos, el menor número de diarreas se obtuvo con el tratamiento donde se adicionó 15 $\mathrm{mL}$ del preparado microbiano/kgPV y el mayor en el tratamiento control, excepto para $E$. coli que, contrario a lo esperado, fue superior en el grupo con $5 \mathrm{~mL} / \mathrm{kg}$ de PV, lo que debe comprobarse en estudios posteriores. 
Tabla 5. Causas y número total de animales con diarreas al suministrar tres dosis del preparado microbiano en cerdos post destete.

\begin{tabular}{|c|c|c|c|c|c|}
\hline $\begin{array}{l}\text { Causas de las } \\
\text { diarreas }\end{array}$ & Tratamientos & $\begin{array}{c}\text { Número de } \\
\text { animales con } \\
\text { diarreas }\end{array}$ & $\begin{array}{c}\% \text { de animales } \\
\text { con diarrea }\end{array}$ & \begin{tabular}{|c|} 
Total de \\
animales con \\
diarrea
\end{tabular} & $\begin{array}{l}\text { EE }( \pm) \\
\text { Signif. }\end{array}$ \\
\hline \multirow{4}{*}{ E. coli } & Control & 48 & $29,45^{b}$ & \multirow{4}{*}{163} & \multirow{4}{*}{$\begin{array}{c}3,39 \\
P<0,0001\end{array}$} \\
\hline & $5 \mathrm{~mL} / \mathrm{kgPV}$ & 72 & $44,17^{a}$ & & \\
\hline & $10 \mathrm{~mL} / \mathrm{kgPV}$ & 31 & $19,02^{c}$ & & \\
\hline & $15 \mathrm{~mL} / \mathrm{kgPV}$ & 12 & $7,36^{d}$ & & \\
\hline \multirow{4}{*}{$\begin{array}{l}\text { Salmo ne lla } \\
\text { spp. }\end{array}$} & Control & 32 & $42,67^{\mathrm{a}}$ & \multirow{4}{*}{75} & \multirow{4}{*}{$\begin{array}{c}5,00 \\
P<0,0001\end{array}$} \\
\hline & $5 \mathrm{~mL} / \mathrm{kgPV}$ & 24 & $32,0^{\mathrm{ab}}$ & & \\
\hline & $10 \mathrm{~mL} / \mathrm{kgPV}$ & 14 & $18,67^{\mathrm{bc}}$ & & \\
\hline & $15 \mathrm{~mL} / \mathrm{kgPV}$ & 5 & $6,67^{c}$ & & \\
\hline \multirow{4}{*}{$\begin{array}{l}\text { Problemas di- } \\
\text { gestivos }\end{array}$} & Control & 248 & $54,63^{\mathrm{a}}$ & \multirow{4}{*}{454} & \multirow{4}{*}{$\begin{array}{c}2,03 \\
P<0,0001\end{array}$} \\
\hline & $5 \mathrm{~mL} / \mathrm{kgPV}$ & 116 & $25,55^{b}$ & & \\
\hline & $10 \mathrm{~mL} / \mathrm{kgPV}$ & 59 & $13,00^{c}$ & & \\
\hline & $15 \mathrm{~mL} / \mathrm{kgPV}$ & 31 & $6,83^{d}$ & & \\
\hline
\end{tabular}

$\mathrm{a}, \mathrm{b}, \mathrm{c}, \mathrm{d}$ letras distintas por columna indican diferencias para $\mathrm{P}<0,05$ (Duncan 1955)

La disminución de las diarreas por procesos infecciosos quizás se relaciona con la producción de sustancias antimicrobianas, como las bacteriocinas y los ácidos orgánicos, que pueden ser producidas por las bacterias lácticas presentes en el preparado microbiano, además de las concentraciones de los metabolitos presentes en este producto, según lo informado por (10). Los ácidos orgánicos (entre ellos el ácido láctico y los de cadena corta) pudieron disminuir el $\mathrm{pH}$ en el intestino que, a su vez, alteraron o inhibieron el crecimiento de microorganismos patógenos (como los coliformes que son sensibles a estos bajos $\mathrm{pH}$ ) y, consecuentemente, favorecieron la eubiosis intestinal $(29,30,31)$. Por su parte, algunas de las especies presentes en el aditivo, como Lactobacillus acidophilus, pueden producir bacteriocinas en altas proporciones con notable efecto contra Salmonella (32) y E. coli (33).

Otros de los mecanismos por los que el preparado ecuatoriano pudo actuar al ser ingerido en la dieta de los cerditos son la competencia por los sitios de adhesión, la adherencia efectiva a las células del epitelio intestinal, la competencia por los nutrientes, el crecimiento microbiano y la alteración del metabolismo microbiano y del animal huésped. Aspectos que se informan en la literatura científica para microorganismos probióticos, por autores como (34), aunque no se conozcan con exactitud. Estos elementos al parecer pudieron influir en la reducción de los problemas digestivos observados en el presente experimento y en la mejora de la digestión y absorción de nutrientes, con efecto en la respuesta productiva.

Al encontrar múltiples efectos beneficiosos producidos por las bacterias lácticas y levaduras del preparado ecuatoriano en los indicadores antes analizados, era de esperar la incidencia de éste en el comportamiento de la mortalidad registrada para cada uno de los grupos tratados. Sin embargo, en la figura 2 se muestra que no hubo diferencias 
estadísticas entre los tratamientos para la mortalidad, aunque biológicamente se obtiene el menor porcentaje con los niveles 10 y $15 \mathrm{~mL} / \mathrm{kgPV}$ y el mayor porcentaje con el control, lo que incide en la relación beneficio costo al tener menor cantidad de animales para la venta. Estos resultados son similares a los observados por autores como $(6,35)$.

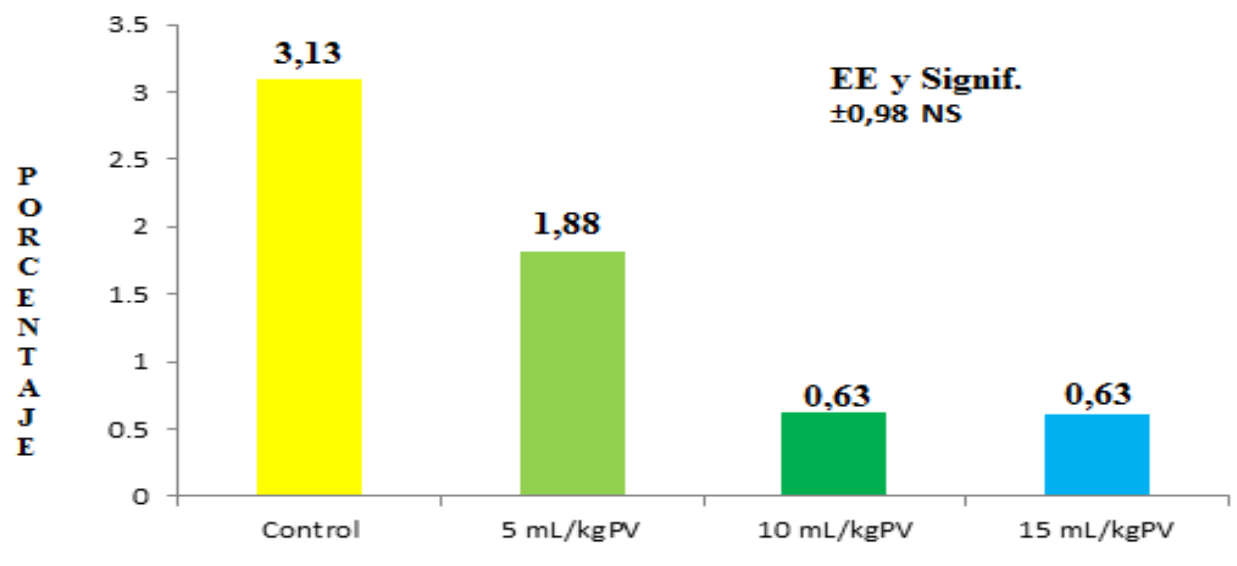

TRATAMIENTOS

Figura 2. Mortalidad de cerditos destetados al incluir en la dieta tres dosis del preparado microbiano.

De forma general, la respuesta productiva y sanitaria de los cerditos en experimentación demuestra que el preparado microbiano empleado produce actividad probiótica. Sus efectos son similares a otros aditivos microbianos puros o mixtos como los informados por $(36,37,38,39)$.

Se concluye que el preparado microbiano del Ecuador mejora la respuesta productiva y sanitaria de cerdos en post-destete y que la adición en la dieta de $15 \mathrm{~mL}$ del preparado microbiano por kilogramo de peso vivo, correspondiente a $6,0 \times 10^{7}$ UFC de BAL y $2,25 \times 10^{6}$ UFC de levaduras, incrementa su eficacia en la respuesta productiva y sanitaria durante la etapa de post destete porcino.

\section{Referencias}

(1) Brooks, P.H., Moran, C.A., Beal, J.D., Demeckova, V. \& Campbell., A. 2001. Liquid feeding for the young piglets. In: M. A. Varley, J. R. Wiseman (eds), The Weaner Pig: Nutrition and Management. CAB International, Wallinford, Oxon. p. 153. DOI: http://dx.doi. org/10.1079/9780851995328.0153.
(2) Williams, I. 2003. Growth of the weaned pig. In: J. R. Pluske, J. V. Le Dividich, M. W. A. Verstegen (eds), Weaning the Pig: Concepts and Consequences, Wageningen Academic Publishers, Wageningen, Netherlands. p. 2527.

(3) Lalles, J., Boudry, G., Favier, C., Le Floc'h, N., Lurona, I., Montagne, L., Oswald, I. P., Pie, S., Piel, C. \& Seve, B. 2004. Gut function and dysfunction in young pigs: physiology. Animal Research 53: 301-316. DOI: http://dx.doi. org/10.1051/animres:2004018.

(4) Cajaeville, C., Brambillasca, S. \& Zumino, P. 2011. Utilización de prebióticos en monogástricos: aspectos fisiológicos y productivos relacionados al uso de subproductos de agroindustrias y de pasturas en lechones Revista Porcicultura Iberoamericana 1:2.

(5) Maron, D., Smith, T. \& Nachman, K. (2013). Restrictions on antimicrobial use in food animal production: an international regulatory and economic survey. Globalization and Health. 9: 1-11. DOI: http://dx.doi. org/10.1186/1744-8603-9-48. 
(6) Faria, D., Torres, K., Campos, D. \& Rosa, P. 2006. Probiotics for broiler chickens in Brazil: systematic review and meta-analysis. Brazilian Journal of Poultry Science. 8: 8997. DOI: http://dx.doi.org/10.1590/s1516$635 \times 2006000200004$.

(7) Guartazaca, L. 2011. Valoración en la alimentación de los lechones post destete con la utilización de dos probióticos. Tesis de Ingeniería. Universidad del Azuay. Cuenca, Ecuador. 62 p.

(8) Gaibor, C. 2012. Comparación de la respuesta biológica de un probiótico comercial vs un antbiótico en las etapas de crecimiento y engorde en porcinos. Tesis de Ingeniería. Escuela Superior Politécnica de Chimborazo. Riobamba, Ecuador. 62 p.

(9) Quemac, M. 2014. Evaluación de tres dosis de probiótico (Rhodopseudomonas spp, Lactobacillus spp, Saccharomyces spp) en la alimentación para el engorde de cerdos. Tesis de Ingeniería. Universidad Estatal Politécnica del Carchi. Tulcán, Ecuador. 48 p.

(10) Díaz, B. 2014. Evaluación de residuos agrícolas post cosecha en ensilajes inoculados con preparados microbianos nativos para alimentación de vacas lecheras en Ecuador. Tesis presentada para la opción de Doctor en Ciencias Veterinarias. Mayabeque, Cuba. 112. p.

(11) NRC. 2012. Nutrient Requirement of Domestic Animals. Nutrient Requirements of Swine. National Academic Press.Washington, District of Columbia, version electronic disponible en disco compacto.

(12) Neogen (2011). Salmonella Shigella Agar (7152). Acumedia. Disponible en: < http:// www.neogen.com/Acumedia/pdf/ProdInfo/7152_Pl.pdf $>$. Consulado, Junio 2015.

(13) Petrifilm 3M. (2010). Placas para recuento de E. coli/Coliform. Disponible en < http:// multimedia.3m.com/mws/media/701951O/ product-instructions-3m-petrifilm-e-coli-co- liform-count-plate.pdf $>$ Consultado, Junio 2015.

(14) InfoStat. 2012. Di Rienzo J., Casanoves F., Balzarini M., Gonzalez L., Tablada, M. \& Robledo, C. Grupo InfoStat, FCA, Universidad Nacional de Córdoba, Argentina. Versión 1.0 para Windows.

(15) Duncan, D.B. (1955). Multiple range and multiple $\mathrm{F}$ tests. Biometrics 11:1.

(16) ComparPro versión 1. 2007. Font H. Noda Aida, Torres Verena, Herrera Magaly, Lizazo D., Sarduy Lucía y Rodríguez Lourdes: Instituto de Ciencia Animal, Dpto Biomatemática.

(17) Kim, E., Hong, H., Hong, N., Choi, K., Hann, Y., Kangwan, N., Chao, Y. \& Hahn, K. 2012. Concentrated Probiotics Improve Inflammatory Bowel Diseases Better that Commercial Concentration ofo Probiotics. Journal of Food and Drug Analysis. 20: 292-295.

(18) Noriega, L., Gueimonde, M., Sánchez, B., Margolles, A. \& de los Reyes, C. 2004. Effect of the adaptation to high bile salts concentrations on glycoside activity, sorvival ta low $\mathrm{pH}$ and cross-resistance to bile salts in Bifidobacterium. International Journal Food Microbiol. 94: 79-86. DOI: http://dx.doi.org/10.1016/j. ijfoodmicro.2004.01.003.

(19) FAO/WHO (Food and Agriculture Organization of the United Nations/World Health Organization). Guidelines for the evaluation of probiotics in food. Report of a joint FAO/ WHO working group on drafting guidelines for the evaluation of probiotics in food. April 30 and May 1. London Ontario, Canadá. Disponible en: < http://www.who.int/foodsafety/fs_management/en/probiotic_guidelines. pdf $>$. Consultado, enero 2015.

(20) Gorbach, S. 1991. Lactobacillus. Probiotics in poultry. The Journal Applied. Poultry Research.14: 750-756. 
(21) Iñiguez, C., Jiménez, R., Vázquez, L., Ramos, G. \& Acedo, E. 2011. Protein-carbohydrate interactions between Lactobacillus salivarius and pig mucins. Journal of Animal Science. 89: 3125-3131. DOI: http://dx.doi.org/10.2527/ jas.2010-2996.

(22) Jurado. H., Ramírez, C. \& Martínez, J. 2013. In vivo evaluation of Lactobacillus platarum as an alternative to antibiotics in piglets. Rev. MVZ Córdova. 18: 3648-3657.

(23) Spanhaak, S., Havenaar, R. \& Schaafsma, G. 1998. The effect of consumption of milk fermented by Lactobacillus casei strain Shirota on the intestinal microflora and immune parameters in humans. European Journal of Clinical Nutrition. 52: 899-907. DOI: http://dx.doi. org/10.1038/sj.ejcn.1600663.

(24) Matsuzaki, T. 1998. Immunomodulation by treatment with Lactobacillus casei strain Shirota. International Journal of Food Microbiology, 41: 133-140. http://dx.doi.org/10.1016/ S0168-1605(98)00046-4.

(25) Boucourt, R., Savón, L. \& Díaz, J. 2002. Efecto de la actividad probiótica de Lactobacillus rhamnosus en indicadores fisiológicos, productivos y de salud de cerdos jóvenes. En: Memorias XVIII Congreso Panamericano de Ciencias Veterinarias (PANVET). 18-22 de noviembre. Palacios de las Convenciones, Ciudad Habana, Cuba. p 135.

(26) Sanz, Y., Collado, M.C. \& Dalmau, J. 2003. Probióticos: criterios de calidad y orientaciones para el consumo. Acta Pediátrica Española. 61: 476-482.

(27) Boyle, R., Robins-Browne, R. \& Tang, M. 2006. Probiotic use in clinical practice: what are the risks? The American Journal Clinical Nutrition. 83: 1256-1264.

(28) Castro, M. \& Rodríguez, F. 2005 Levaduras: probióticos y prebióticos que mejoran la producción animal. Revista Corpoica. 6: 26-38.
(29) Fuller, R. 1992. Chapter 1. History and development of probiotic. En: Probiotics. The Scientific Basic. Fuller, R. (ed.). Chapman \& Hall, London, UK. p. 4. DOI: http://dx.doi. org/10.1007/978-94-011-2364-8.

(30) Sadler, M., Strain, J. \& Caballero, B. 1998. Probiotics and prebiotics. Definition and role. En: Encyclopedia of Human Nutrition. Academic Press (ed.). London \& New York. p. 1633.

(31) Gauthier, R. 2002. Nuevas alternativas en terapéutica aviar. En: XVIII Congreso Latinoamericano de Avicultura. 7-10 de octubre. Santa Cruz, Bolivia. p. 156.

(32) Tahara, T. \& Kanatani, K. 1997. Isolation and partial aminoacid sequence of bacteriocins produced by Lactobacillus acidophilus. Bioscience, Biotechnology and Biochemistry. 61: 884-886. DOI: http://dx.doi.org/10.1271/ bbb.61.884.

(33) Gillor, O., Etzion, A. \& Riley, M. 2008. The dual role of bacteriocins as anti- and probiotics. Applied Microbiology Biotechnology. 81: 591-606. DOI: http://dx.doi.org/10.1007/ s00253-008-1726-5.

(34) Chukeatirote, E. 2003. Potencial use of probiotics. Songklanakarin Journal Science Technological. 25: 275-282.

(35) García, R., Hernández, K., Kawas, J., Salinas, J., Vega, A., Ruiloba, M. \& Fimbres, H. 2014. Efecto de nucleótidos y péptidos de Saccharomyces cerevisiae (NUTRO) en la alimentación de cerdos post destete. Revista Científica Facultad de Ciencias Veterinarias de la Universidad de Zulia. 24: 29-37.

(36) Roján, L. 2009. Efecto de un producto biológicamente activo (Vitafert) en indicadores productivos y de salud en preceba porcina. Tesis de Maestría. Instituto de Ciencia Animal. Cuba. 76 p.

(37) Wang, Y., Cho, J., Chen, Y., Yoo, J., Huang, Y., Kim, H. \& Kim, I. 2009. The effect of pro- 
biotic BioPlus $2 \mathrm{~B}^{\circledR}$ on growth performance, dry matter and nitrogen digestibility and slurry noxious gas emission in growing pigs. Livestock Science. 120: 35-42. DOI: http://dx.doi. org/10.1016/j.livsci.2008.04.018

(38) Trckova, M., Faldyna, M., Alexa, P., Sramkova. Z., Gopfert, E., Kumprechtova, D., Auclair, E. \& Auclair, R. 2014. The effects of live yeast Saccharomyces cerevisiae on postweaning diarrhea, immune response, and growth per- formance in weaned piglets. Journal of Animal Science. 92: 767-774. DOI: http://dx.doi. org/10.2527/jas.2013-6793.

(39) Liu, H., Zhang, J., Zhang, S., Yang, F., Thacker, PA. \& Zhang, G. 2014. Oral administration of L. fermentum 15007 favors intestinal development and alters the intestinal microbiota in formula-fed piglets. Journal of Agricultural and Food Chemistry. 62: 860-866. DOI: http://dx. doi.org/10.1021/jf403288r. 